\title{
Hybridized CutFEM for Elliptic Interface Problems
}

\author{
Erik Burman, Daniel Elfverson, Peter Hansbo, \\ Mats G. Larson and Karl Larsson
}

\begin{abstract}
We design and analyze a hybridized cut finite element method for elliptic interface problems. In this method very general meshes can be coupled over internal unfitted interfaces, through a skeletal variable, using a Nitsche type approach. We discuss how optimal error estimates for the method are obtained using the tools of cut finite element methods and prove a condition number estimate for the Schur complement. Finally, we present illustrating numerical examples.
\end{abstract}

\section{Introduction}

The solution of heterogeneous problems, for instance problems where some physical parameter has important variation within the computational domain, remains a challenging problem in computational science. Indeed special care must be taken in the design of methods to ensure that efficiency and accuracy do not degenerate in the presence of high contrast inclusions. An additional layer of complexity is added if the internal structure, e.g. the position of the inclusion, is one of the problem unknowns and domain boundaries or internal interfaces must be modified during the computation. In this situation it is an advantage if remeshing of the domain can be avoided, while the equations still are solved accurately on a variety of configurations [5,34]. When one interface separates two computational domains and the problem size is moderate it is reasonable to use a monolithic solution strategy. However, as the number of inclusions increases it becomes advantageous to resort to domain decomposition so that the problem can be solved in parallel.

Recently there has been a surge of activity in the design and analysis of hybridized methods, that is nonconforming methods that have different approximating polynomials defined in the bulk of the elements and on the skeleton of the computational mesh. The skeleton variable plays the role of a mortar variable, either for a Neumann or a Dirichlet trace. Typically the interior degrees of freedom of each cell can easily be eliminated using static condensation, thereby reducing the size of the linear system. This is particularly appealing for high order approximation methods where each volume element hosts a relatively large number of degrees of freedom. An important feature of hybridized methods is that they allow for fairly general element shapes and there exists an important literature on methods defined on general polygonal/polyhedral meshes. Examples of such methods include the Hybridized Discontinuous Galerkin Method [15], the Virtual Element Method [1] and the Hybridized High Order method [16]. In many cases these methods are closely related (see [14 and references therein). 
In this contribution we design a hybridized finite element method within the cut finite element method (CutFEM) paradigm, see [4,6]. This means that the computational mesh is independent of the geometry and internal interfaces, for example the computational mesh can remain completely structured. If the underlying problem has some special structure dividing the computational domain in subdomains, for instance defined by grains with a specific permeability or microstructure, the domain boundaries are allowed to cut through the background mesh in a close to arbitrary fashion. By including boundary or interface defining terms in the variational formulation the method essentially eliminates the meshing problem. This is particularly convenient in shape optimization problems or inverse identification problems, where the position of the interfaces change during an optimization process. The introduction of a skeletal variable makes it possible to eliminate internal degrees of freedom in a parallel fashion so that the linear system can be solved by iterating on the Schur complement. Optimal stability and accuracy is obtained using the tools developed within the CutFEM paradigm. Below we will also show that the resulting Schur complement has a condition number that is bounded independently of the mesh interface intersection.

Brief Review of Cut Finite Elements. CutFEMs were originally introduced by Hansbo and Hansbo 22 as an alternative to extended finite element methods, using Nitsche's method as mortaring over an unfitted interface. The ideas were extended to composite meshes, Chimera-style, by Hansbo, Hansbo and Larson [23] and then to fictitious domain problems by Burman and Hansbo [6]. In a parallel development [33], Olshanski, Reusken and Grande developed a cut finite element method for the discretization of PDEs on surfaces using the trace of the (discrete) surface on a finite element bulk mesh as the computational mesh. Additional stability can be obtained by adding suitable stabilization terms $[3,7,11,21,28]$ and the methods are then comparable to standard finite element methods on meshed geometries, both with respect to conditioning and accuracy. Further developments include techniques for the handling of curved interfaces or boundaries [8, 29], PDEs on composite surfaces [24] and transport problems in fractured mixed dimensional domains [10]. For an overview of the ideas behind the CutFEM paradigm see [4] and for a collection of essays giving a snapshot of the state of the art we refer to $[2]$.

New Contributions. We develop a hybridized CutFEM for an elliptic model problem with piecewise constant coefficients defined on a partition of the domain. The union of the boundaries of the subdomains is called the skeleton and hybridization consists of adding a solution field representing the solution on the skeleton. In the proposed method each subdomain of the bulk and each component of the skeleton is equipped with its own finite element mesh and space. The mesh may be constructed using a cut technique or a standard mesh. The hybridization leads to a convenient formulation which also naturally facilitates elimination of the bulk degrees of freedom using a Schur complement formulation.

We develop a stability and error analysis where we in particular show that optimal order a priori error estimates holds for both and that the condition number of the Schur complement is $O\left(d^{-1} h^{-1}\right)$ where $d$ is the diameter of the subdomain. We cover very general choices of the subdomains and one instance of our method and analysis is a $p$ based discontinuous Galerkin method on polygonal elements. 

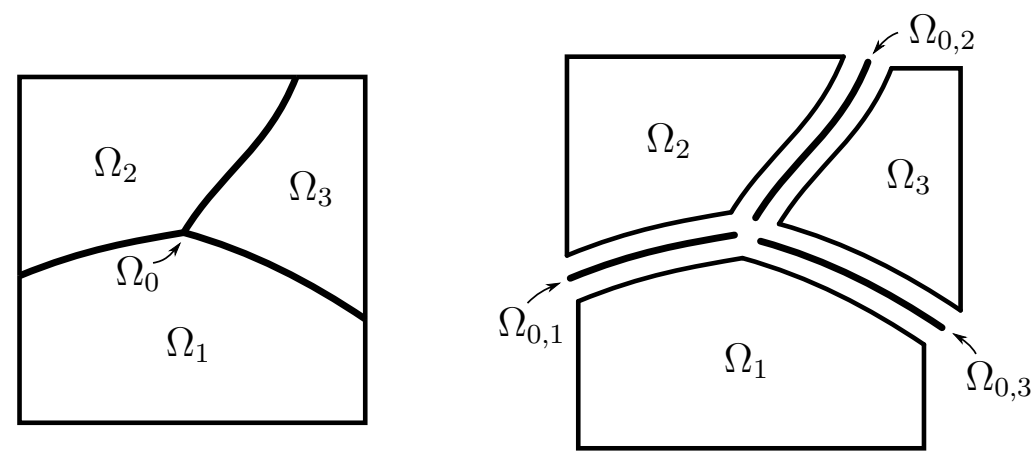

Figure 1: Schematic illustration of a partition of the domain $\Omega$ into a set of subdomains $\left\{\Omega_{i}\right\}_{i=1}^{N}$ and of the skeleton $\Omega_{0}$ with its partition into a set of skeleton subdomains $\left\{\Omega_{0, i}\right\}_{i=1}^{N_{0}}$.

Earlier Work. Hybridized methods are commonly used and many versions have been proposed, notably in the setting of discontinuous Galerkin methods; for an overview, see Cockburn et al. [15. The particular version we employ here was first proposed using meshed subdomains by Egger [17], who later extended it to incompressible flow [19, 20] and convection-diffusion problems [18]; cf. also Könnö and Stenberg [27] where it was employed for solving the Brinkman problem. Independently, the same approach has been proposed by Oikawa and Kikuchi [32] and further developed by Miyashita and Saito [31].

Outline. In Section 2 we formulate the hybridized CutFEM. In Section 3 we derive stability and optimal order error estimates. In Section 4 we eliminate the bulk fields using the Schur complement and we derive a bound for the condition number of the Schur complement. In Section 5 we present numerical results.

\section{The Hybridzed Cut Finite Element Method}

\subsection{The Model Problem}

Domain. In hybridized methods the domain $\Omega$ is partitioned into a set of subdomains $\left\{\Omega_{i}\right\}_{i=1}^{N}$ and the interfaces between between the subdomains constitute a skeleton $\Omega_{0}$, see the illustration in Figure 1. Formally we assume the following:

- Let $\Omega \subset \mathbb{R}^{d}$, with $d=2$ or 3 , be a domain with piecewise smooth Lipschitz boundary $\partial \Omega$. Recall that $\partial \Omega$ is piecewise smooth if it is the union of a finite number of smooth $d-1$ dimensional manifolds with boundary and Lipschitz if there is a finite set of local coordinate systems in which the boundary can be described as a Lipschitz function.

- Let $\left\{\Omega_{i}\right\}_{i=1}^{N}$ be a partition of $\Omega$ into $N$ subdomains $\Omega_{i}$ with piecewise smooth Lipschitz boundaries $\partial \Omega_{i}$.

- Let $\Omega_{0}=\cup_{i=1}^{N} \partial \Omega_{i} \backslash \partial \Omega$ be the skeleton of the partition. Note that there is a partition of $\Omega_{0}$ into smooth $d-1$ manifolds with boundary

$$
\Omega_{0}=\cup_{i, j=1}^{N} \partial \Omega_{i} \cap \partial \Omega_{j}=\cup_{i=1}^{N_{0}} \Omega_{0, i}
$$

where $N_{0}$ is the number of nonempty intersections $\partial \Omega_{i} \cap \partial \Omega_{j}$. 
Model Problem. We consider the following hybridized formulation of the Poisson problem: find $u_{0}: \Omega_{0} \rightarrow \mathbb{R}$ and for $i=1, \ldots, N, u_{i}: \Omega_{i} \rightarrow \mathbb{R}$ such that

$$
\begin{aligned}
-\nabla \cdot a_{i} \nabla u_{i} & =f_{i} & & \text { in } \Omega_{i} \\
\llbracket n \cdot a \nabla u \rrbracket & =0 & & \text { on } \Omega_{0} \\
{[u]_{i} } & =0 & & \text { on } \partial \Omega_{i} \cap \Omega_{0} \\
u_{i} & =0 & & \text { on } \partial \Omega_{i} \cap \partial \Omega
\end{aligned}
$$

Here $a_{i}, i=0, \ldots, N$, are positive constants and the jumps operators are defined by

$$
\left.[v]\right|_{\partial \Omega_{i} \cap \Omega_{0}}=v_{i}-v_{0},\left.\quad \llbracket n \cdot a \nabla v \rrbracket\right|_{\partial \Omega_{i} \cap \partial \Omega_{j}}=n_{i} \cdot a_{i} \nabla v_{i}+n_{j} \cdot a_{j} \nabla v_{j}
$$

where $n_{i}$ is the exterior unit normal to $\Omega_{i}$.

This problem is well posed, with exact solution $u \in H_{0}^{1}(\Omega)$ such that $u_{i}=\left.u\right|_{\Omega_{i}}$, $i=0, \ldots, N$. Below we will assume the additional regularity $u_{i} \in H^{\frac{3}{2}}\left(\Omega_{i}\right)$. We define the spaces

$$
V_{0}:=\bigoplus_{i=1}^{N_{0}} L^{2}\left(\Omega_{0, i}\right), \quad V_{1, N}:=\bigoplus_{i=1}^{N} H^{\frac{3}{2}}\left(\Omega_{i}\right)
$$

and the global space

$$
W:=V_{0} \oplus V_{1, N} .
$$

We will use the notation $(\cdot, \cdot)_{X}$ for the $L^{2}$-scalar product over $X \subset \mathbb{R}^{s}$ where either $s=d$ or $s=d-1$. The associated norm will be denoted $\|\cdot\|_{X}$. We will also use the following weighted $L^{2}$-norm, $\|v\|_{\omega, a}^{2}=(a v, v)_{\omega}$, for $a \in L^{\infty}(\omega)$ with $a>0$.

Remark 2.1. We note that solutions to certain problems of the form (2.2.2.5) may have regularity arbitrarily close to $H^{1}(\Omega)$, see [26] where a problem with intersecting interfaces and discontinuous coefficients are considered. Thus (2.7) does not hold in general. For smooth interfaces the assumption holds and of course for the situation when the coefficient is globally constant. Both of these cases are of practical importance since hybridization is used to conveniently enable elimination of the subdomain unknowns using a Schur complement procedure, see Section 3. Deriving error bounds with lower regularity assumptions requires a more involved analysis and we refer to the techniques developed in [9] for CutFEM approximations of boundary value problems with mixed Dirichlet/Neumann boundary conditions and minimal regularity requirements.

\subsection{The Method}

Finite Element Spaces. On each subdomain $\Omega_{i}, 1 \leq i \leq N$, and on each skeleton subdomain $\Omega_{0, i}, 1 \leq i \leq N_{0}$, we construct a separate finite element space.

- Let $O \in\left\{\Omega_{0, i}\right\}_{i=1}^{N_{0}} \cup\left\{\Omega_{i}\right\}_{i=1}^{N}$, i.e., $O$ is either one of the $d$ dimensional bulk subdomains $\Omega_{i}$ or one of the $d-1$ dimensional components of the skeleton $\Omega_{0}$.

- For each $O$ there is a $d$ dimensional domain $U_{O}$ such that $O \subseteq U_{O}$. For each $h \in$ $\left(0, h_{\max , i}\right]$ there is a $d$ dimensional quasiuniform mesh $\mathcal{T}_{h}\left(U_{O}\right)$ on $U_{O}$ with mesh parameter $h$, i.e. $\mathcal{T}_{h}\left(U_{O}\right)$ is a partition of $U_{O}$ into shape regular elements $T$ with diameter $h_{T}$ and $h_{T} \sim h$ uniformly. The active mesh is defined by

$$
\mathcal{T}_{h, O}=\left\{T \in \mathcal{T}_{h}\left(U_{O}\right): T \cap O \neq \emptyset\right\}
$$

which covers $O$ but is not required to perfectly match $O$. 
- Let $V_{h, O}$ be a finite dimensional space consisting of continuous piecewise polynomial functions defined on $\mathcal{T}_{h, O}$ such that that there is an interpolation operator $\pi_{h, i}$ : $H^{1}(O) \rightarrow V_{h, i}$, which satisfy the approximation property

$$
\left\|v-\pi_{h} v\right\|_{H^{m}\left(\mathcal{T}_{h, O}\right)} \lesssim h^{s-m}\|v\|_{H^{s}(O)} \quad m=0,1, \quad m \leq s \leq p+1
$$

where $p+1$ is the approximation order of $V_{h, O}$.

- For $O \in\left\{\Omega_{i}\right\}_{i=1}^{N}$ we use the simplified notation

$$
\mathcal{T}_{h, \Omega_{i}}=\mathcal{T}_{h, i}, \quad V_{h, \Omega_{i}}=V_{h, i}
$$

we also define

and for $O \in\left\{\Omega_{0, i}\right\}_{i=1}^{N_{0}}$,

$$
\mathcal{T}_{h, 0}=\sqcup_{i=1}^{N_{0}} \mathcal{T}_{h, 0, i}
$$

$$
\mathcal{T}_{h, \Omega_{0, i}}=\mathcal{T}_{h, 0, i}, \quad V_{h, \Omega_{0, i}}=V_{h, 0, i}
$$

- Define the finite element spaces

$$
V_{h, 0}=\bigoplus_{i=1}^{N_{0}} V_{h, 0, i}, \quad V_{h, 1, N}=\bigoplus_{i=1}^{N} V_{h, i}
$$

and

$$
W_{h}=V_{h, 0} \oplus V_{h, 1, N}
$$

See Figures 3 and 5 in Section 4 for illustrations of the construction of the mesh.

Remark 2.2. Here we are using restrictions of $d$ dimensional spaces to the $d-1$ dimensional skeleton in the spirit of CutFEM, see [7, 33], where similar ideas are used to solve the Laplace-Beltrami problem on a codimension one surface embedded in d dimensional mesh. We may also use a standard $d-1$ dimensional mesh on each of the components $\Omega_{0, i}$ of the skeleton. Note that these meshes does not need to match on the interfaces $\bar{\Omega}_{0, i} \cap \bar{\Omega}_{0, j}$ between two components of the skeleton. Our analysis readily extends to this situation as well.

Stabilization Forms. We here define a number of abstract properties, which we assume our stabilization forms satisfy. In Section 3.3 below we construct forms that fulfill these properties. For $i=0, \ldots, N$ there are symmetric bilinear forms $s_{h, i}$, on $V_{h, i}+H^{p+1}\left(\Omega_{i}\right)$, with associated semi norm $\|\cdot\|_{s_{h, i}}$ such that:

- For $v \in H^{p+1}\left(\Omega_{i}\right)$ it holds

$$
s_{h, i}(v, w)=0 \quad \forall w \in V_{h, i}
$$

and

$$
\left\|v-\pi_{h} v\right\|_{s_{h, i}} \lesssim h^{p}\|v\|_{H^{s}\left(\Omega_{i}\right)}
$$

- For $v \in V_{h, 0, i}, i=1, \ldots, N_{0}$ there are constants such that

$$
h^{-1}\|v\|_{\mathcal{T}_{h, 0, i}}^{2} \sim\|v\|_{\Omega_{0, i}}^{2}+\|v\|_{s_{h, 0, i}}^{2} \quad v \in V_{h, 0, i}
$$

- For $v \in V_{h, i}, i=1, \ldots, N$, there is a constant such that

$$
\|v\|_{\mathcal{T}_{h, i}}^{2} \lesssim\|v\|_{\Omega_{i}}^{2}+\|v\|_{s_{h, i}}^{2} \quad v \in V_{h, i}
$$

- For $v \in V_{h, i}, i=1, \ldots, N$, there is a constant such that

$$
h\|\nabla v\|_{\partial \Omega_{i}, a_{i}}^{2} \lesssim\|\nabla v\|_{\Omega_{i}, a_{i}}^{2}+\|v\|_{s_{h, i}}^{2} \quad v \in V_{h, i}
$$


Derivation of the Method. Multiplying (2.2) by a test function $v$, integrating by parts over $\Omega_{i}$, and using the convention $u_{0}=0$ on $\partial \Omega$ we obtain

$$
\begin{aligned}
\sum_{i=1}^{N}\left(f_{i}, v_{i}\right)_{\Omega}= & \sum_{i=1}^{N}-\left(\nabla \cdot a_{i} \nabla u_{i}, v_{i}\right)_{\Omega_{i}} \\
= & \sum_{i=1}^{N}\left(a_{i} \nabla u_{i}, \nabla v_{i}\right)_{\Omega_{i}}-\left(n_{i} \cdot a_{i} \nabla u_{i}, v_{i}\right)_{\partial \Omega_{i}} \\
= & \sum_{i=1}^{N}\left(a_{i} \nabla u_{i}, \nabla v_{i}\right)_{\Omega_{i}}-\left(n_{i} \cdot a_{i} \nabla u_{i}, v_{i}\right)_{\partial \Omega_{i}} \\
& \quad-\left(u_{i}-u_{0}, n \cdot a_{i} \nabla v_{i}\right)_{\partial \Omega_{i}}+\left(\beta h^{-1} a_{i}\left(u_{i}-u_{0}\right), v_{i}\right)_{\partial \Omega_{i}} \\
= & \sum_{i=1}^{N}\left(a_{i} \nabla u_{i}, \nabla v_{i}\right)_{\Omega_{i}}+s_{h, i}\left(u_{i}, v_{i}\right)-\left(n_{i} \cdot a_{i} \nabla u_{i}, v_{i}-v_{0}\right)_{\partial \Omega_{i}} \\
& \quad-\left(u_{i}-u_{0}, n_{i} \cdot a_{i} \nabla v_{i}\right)_{\partial \Omega_{i}}+\left(\beta h^{-1} a_{i}\left(u_{i}-u_{0}\right),\left(v_{i}-v_{0}\right)\right)_{\partial \Omega_{i}}
\end{aligned}
$$

where we added terms which are zero for the exact solution $u$ with the purpose of obtaining a stable symmetric formulation. More precisely, in (2.24) we used (2.4) to add terms involving $[u]_{i}=u_{i}-u_{0}=0$ and in $(2.25)$ we used $(2.3)$ and the identity

$$
0=\left(\llbracket n \cdot a \nabla u \rrbracket, v_{0}\right)_{\Omega_{0}}=\sum_{i=1}^{N}\left(n_{i} \cdot a_{i} \nabla u_{i}, v_{0}\right)_{\partial \Omega_{i}}
$$

since $v_{0}=0$ on $\partial \Omega$. Finally, we added the stabilization form $s_{h, i}$ using consistency (2.16).

Definition of the Method. Find $u_{h} \in W_{h}$ such that

$$
A_{h}\left(u_{h}, v\right)=l_{h}(v) \quad \forall v \in W_{h}
$$

where $W_{h}$ is defined in 2.15 and

$$
\begin{aligned}
A_{h}(v, w)=\sum_{i=1}^{N} & \left(a_{i} \nabla v_{i}, \nabla w_{i}\right)_{\Omega_{i}}+s_{h, i}\left(v_{i}, w_{i}\right) \\
& \quad-\left(n_{i} \cdot a_{i} \nabla v_{i},[w]_{i}\right)_{\partial \Omega_{i}}-\left([v]_{i}, n_{i} \cdot a_{i} \nabla w_{i}\right)_{\partial \Omega_{i}} \\
& +\left(\beta h_{i}^{-1} a_{i}[v]_{i},[w]_{i}\right)_{\partial \Omega_{i}} \\
& +s_{h, 0}\left(v_{0}, v_{0}\right) \\
l_{h}(v)= & \sum_{i=1}^{N}\left(f_{i}, v_{i}\right)_{\Omega_{i}}
\end{aligned}
$$

Remark 2.3. We consider the partition $\left\{\Omega_{i}\right\}_{i=1}^{N}$ of $\Omega$ as being fixed for simplicity only. In fact we may also allow the number of subdomains $N$ to increase during refinement. We then obtain a version of the polygonal finite element method, where each polygonal finite element is equipped with a mesh and piecewise continuous polynomials of degree $p$, and not only polynomials of order $p$ which in general is the case in polygonal finite elements. In order to guarantee that coercivity holds under refinement of the partition we essentially need the inverse inequality (2.20) to hold with a uniform constant for all 
subdomains that occur during the refinement of the partition. Therefore we need to assume some additional shape regularity of the subdomains. For instance, we may assume that for each $\Omega_{j}$ there is partition $\mathcal{S}_{j}$ of $\Omega_{i}$ into simplicies $S$ and a constant such that for all $S \in \mathcal{S}_{j}$ with $|\partial S \cap \partial \Omega|>0$ it holds

$$
h_{i} \lesssim \frac{|S|}{\left|\partial S \cap \partial \Omega_{i}\right|}
$$

where the mesh parameter $h_{i} \in\left(0, h_{0, i}\right]$. Then (2.20) holds uniformly over all partitions $\left\{\Omega_{j}\right\}_{j=1}^{N}$ of $\Omega$, with corresponding meshes $\mathcal{T}_{h, i}$.

The corresponding condition for polygonal finite elements is

$$
\operatorname{diam}\left(\Omega_{i}\right) \lesssim \frac{|S|}{\left|\partial S \cap \partial \Omega_{i}\right|}
$$

see Assumption 30, Section 4.3 in [12], and since we may always assume that $h_{i} \lesssim$ $\operatorname{diam}\left(\Omega_{i}\right)$ we conclude that (2.34) is weaker than (2.35) and thus more complex subdomains may be employed when finer meshes are used.

\section{$3 \quad$ Error Estimates}

The error estimates for our methods are obtained in a similar fashion as those proven in [17] and we will focus here on how robustness and optimal estimates are obtained in the framework of cut elements.

\subsection{Properties of $A_{h}$}

Define the energy norm

$$
\|\| v\|\|_{h}^{2}=\sum_{i=1}^{N}\left\|\nabla v_{i}\right\|_{\Omega_{i}, a_{i}}^{2}+h\left\|\nabla v_{i}\right\|_{\partial \Omega_{i}, a_{i}}^{2}+h^{-1}\left\|[v]_{i}\right\|_{\partial \Omega_{i}, a_{i}}^{2}
$$

Continuity. It follows directly from the Cauchy-Schwarz inequality that $A_{h}$ is continuous

$$
A_{h}(v, w) \lesssim\left\|\left|\|\|_{h}\right|\right\| w \|_{h} \quad v, w \in W+W_{h}
$$

Coercivity. For $\beta$ large enough $A_{h}$ is coercive

$$
\|v\|_{h}^{2} \lesssim A_{h}(v, v) \quad v \in W_{h}
$$


Proof of (3.3). Using standard arguments and the inverse estimate 2.20) we obtain

$$
\begin{aligned}
A_{h}(v, v)= & \sum_{i=1}^{N}\left(a_{i} \nabla v_{i}, \nabla v_{i}\right)_{\Omega_{i}}+s_{h, i}(v, w)-2\left(n_{i} \cdot a_{i} \nabla v_{i},\left[v_{i}\right]\right)_{\partial \Omega_{i}} \\
& +\beta h^{-1}\left\|[v]_{i}\right\|_{\partial \Omega_{i}, a_{i}}^{2} \\
\gtrsim & \sum_{i=1}^{N}\left\|\nabla v_{i}\right\|_{\Omega_{i}, a_{i}}^{2}+\|v\|_{s_{h, i}}^{2}-2 h^{1 / 2}\left\|\nabla v_{i}\right\|_{\partial \Omega_{i}, a_{i}} h^{-1 / 2}\left\|\left[v_{i}\right]\right\|_{\partial \Omega_{i}, a_{i}} \\
& +\beta h^{-1}\left\|[v]_{i}\right\|_{\partial \Omega_{i}, a_{i}}^{2} \\
\gtrsim & \sum_{i=1}^{N}\left(\left\|\nabla v_{i}\right\|_{\Omega_{i}, a_{i}}^{2}+\|v\|_{s_{h, i}}^{2}-\delta h\left\|\nabla v_{i}\right\|_{\partial \Omega_{i}, a_{i}}^{2}\right) \\
& \quad+\left(\beta-\delta^{-1}\right) h^{-1}\left\|[v]_{i}\right\|_{\partial \Omega_{i}, a_{i}}^{2} \\
\gtrsim & \sum_{i=1}^{N}\left(1-\delta C_{1}\right)\left(\left\|\nabla v_{i}\right\|_{\Omega_{i}, a_{i}}^{2}+\|v\|_{s_{h, i}}^{2}\right)+\left(\beta-\delta^{-1}\right) h^{-1}\left\|[v]_{i}\right\|_{\partial \Omega_{i}, a_{i}}^{2}
\end{aligned}
$$

for $\delta>0$ sufficiently small. Here $C_{1}$ is the hidden constant in 2.20).

Poincaré Inequality. Let $\phi$ be the solution to the dual problem

$$
-\nabla \cdot a \nabla \phi=\psi \text { in } \Omega, \quad \phi=0 \text { on } \partial \Omega
$$

with $\psi \in L^{2}(\Omega)$, and assume that

$$
\sum_{i=1}^{N}\left\|\phi_{i}\right\|_{H^{3 / 2}\left(\Omega_{i}\right)}^{2} \lesssim\|\psi\|_{\Omega}^{2}
$$

Under the regularity assumption 3.12 the following Poincaré estimate holds

$$
\left(\min _{1 \leq i \leq N} d_{\Omega_{i}}\right) h^{-1}\left\|v_{0}\right\|_{\mathcal{T}_{h, 0}}^{2}+\sum_{i=1}^{N}\left\|v_{i}\right\|_{\mathcal{T}_{h, i}}^{2} \lesssim\|\| v \|_{h}^{2} \quad v \in W_{h}
$$

where $d_{\Omega_{i}}$ is the diameter of $\Omega_{i}$. This estimate in particular shows that $\|\mid \cdot\| \|_{h}$ is a norm on $W_{h}$.

Proof of (3.13). Consider first the estimation of the bulk subdomain contributions we first note that using 2.19) we have

$$
\sum_{i=1}^{N}\|v\|_{\mathcal{T}_{h, i}}^{2} \lesssim \sum_{i=0}^{N}\|v\|_{\Omega_{i}}^{2}+\|v\|_{s_{h, i}}^{2}
$$


Next to estimate the term $\sum_{i=1}^{N}\|v\|_{\Omega_{i}}^{2}$ we multiply the dual problem 3.11 by $v \in W_{h}$ and using partial integration on each $\Omega_{i}$ we obtain

$$
\begin{aligned}
\sum_{i=1}^{N}\left(v_{i}, \psi_{i}\right)_{\Omega_{i}} & =\sum_{i=1}^{N}\left(a_{i} \nabla v_{i}, \nabla \phi_{i}\right)_{\Omega_{i}}-\left(v_{i}, n_{i} \cdot a_{i} \nabla \phi_{i}\right)_{\partial \Omega_{i}} \\
& =\sum_{i=1}^{N}\left(a_{i} \nabla v_{i}, \nabla \phi_{i}\right)_{\Omega_{i}}-\left([v]_{i}, n_{i} \cdot a_{i} \nabla \phi_{i}\right)_{\partial \Omega_{i}}-\left(v_{0}, n_{i} \cdot a_{i} \nabla \phi_{i}\right)_{\partial \Omega_{i}} \\
& =\sum_{i=1}^{N}\left(a_{i} \nabla v_{i}, \nabla \phi_{i}\right)_{\Omega_{i}}-\left([v]_{i}, n_{i} \cdot a_{i} \nabla \phi_{i}\right)_{\partial \Omega_{i}}-(v_{0}, \underbrace{\llbracket n \cdot a \nabla \phi \rrbracket}_{=0})_{\Omega_{0}} \\
& \lesssim \sum_{i=1}^{N}\left\|\nabla v_{i}\right\|_{\Omega_{i}, a_{i}}\left\|\nabla \phi_{i}\right\|_{\Omega_{i}, a_{i}}+h^{-1 / 2}\left\|[v]_{i}\right\|_{\partial \Omega_{i}, a_{i}} h^{1 / 2}\left\|\nabla \phi_{i}\right\|_{\partial \Omega_{i}, a_{i}} \\
& \lesssim\|v\| \|_{h} \underbrace{\left(\sum_{i=1}^{N}\|\nabla \phi\|_{\Omega_{i}, a_{i}}^{2}+h\|\phi\|_{H^{3 / 2}\left(\Omega_{i}\right), a_{i}}^{2}\right)^{1 / 2}}_{\lesssim\|\psi\|_{\Omega}} \\
& \lesssim\|v\|\left\|_{h}\right\| \psi \|_{\Omega}
\end{aligned}
$$

where in (3.16) we added and subtracted $v_{0}$ in the boundary terms; in (3.17) we used the fact that $v_{0}=0$ on $\partial \Omega$; in (3.18) we used the Cauchy-Schwarz inequality and the fact that $\llbracket n \cdot a \nabla \phi \rrbracket=0$ on $\Omega_{0}$, in (3.19) we used the definition (3.1) of the energy norm and a trace inequality for $\phi$; and finally in $(3.20)$ we used the regularity assumption (3.12). Thus we obtain

$$
\sum_{i=1}^{N}\left\|v_{i}\right\|_{\mathcal{T}_{h, i}}^{2} \lesssim\|v\|_{h}^{2}
$$

Next using the trace inequality

$$
\|v\|_{\partial \Omega_{i}}^{2} \lesssim d_{\Omega_{i}}^{-1}\left\|v_{i}\right\|_{\Omega_{i}}^{2}+d_{\Omega_{i}}\left\|\nabla v_{i}\right\|_{\Omega_{i}}^{2}
$$

we have that

$$
\begin{aligned}
\left\|v_{0}\right\|_{\Omega_{0}}^{2} & \lesssim \sum_{i=1}^{N}\left\|[v]_{i}\right\|_{\partial \Omega_{i}}^{2}+\left\|v_{i}\right\|_{\partial \Omega_{i}}^{2} \\
& \lesssim \sum_{i=1}^{N}\left\|[v]_{i}\right\|^{2}+d_{\Omega_{i}}^{-1}\left\|v_{i}\right\|_{\Omega_{i}}^{2}+d_{\Omega_{i}}\left\|\nabla v_{i}\right\|_{\Omega_{i}}^{2} \\
& \lesssim\left(\min _{1 \leq i \leq N} d_{\Omega_{i}}\right)^{-1}\|v\|_{h}^{2}
\end{aligned}
$$

and thus by 2.18 we obtain

$$
h^{-1}\left\|v_{0}\right\|_{\mathcal{T}_{h, 0}}^{2} \lesssim\left\|v_{0}\right\|_{\Omega_{0}}^{2}+\left\|v_{0}\right\|_{s_{h, 0}}^{2} \lesssim\left(1+\left(\min _{1 \leq i \leq N} d_{\Omega_{i}}\right)^{-1}\right)\|\mid\| \|_{h}^{2}
$$

which concludes the proof since $\left(\min _{1 \leq i \leq N} d_{\Omega_{i}}\right)^{-1} \lesssim 1$.

Note in particular that we have the estimate

$$
\left(\min _{1 \leq i \leq N} d_{\Omega_{i}}\right) h^{-1}\left\|v_{0}\right\|_{\mathcal{T}_{h, 0}}^{2} \lesssim\left\|v_{0}+w\right\|_{h}
$$

for all $w \in V_{h, 1, N}=\oplus_{i=1}^{N} V_{h, i}$. 


\subsection{Interpolation Operator}

\section{Definition of the Interpolation Operator.}

- In order to handle both the $d$ dimensional components and the $d-1$ dimensional components at the same time we let $O \in\left\{\Omega_{0, i}\right\}_{i=1}^{N_{0}} \cup\left\{\Omega_{i}\right\}_{i=1}^{N}$, i.e., $O$ is either one of the $d$ dimensional bulk domains $\Omega_{i}$ or one of the $d-1$ dimensional components of the skeleton $\Omega_{0}$.

- There is an extension operator $E_{O}: H^{s}(O) \rightarrow U_{\delta}(O)$ such that

$$
\|E v\|_{H^{s}\left(U_{\delta}(O)\right)} \lesssim \delta^{1 / 2}\|v\|_{H^{s}(O)}
$$

where $U_{\delta}(O)=\cup_{x \in O} B_{\delta}(x)$, with $B_{\delta}(x)$ denoting a $d$ dimensional ball with radius $\delta>0$ centered in $x$. Note that this means that $U_{\delta}(O)$ is always a $d$ dimensional tubular neighborhood of $O$.

- Let $\mathcal{T}_{h}(O)$ be the mesh associated with $O$ and $V_{h}(O)$ the correponding finite element space. Let $\pi_{h, O, C l}: L^{2}\left(\mathcal{T}_{h}(O)\right) \rightarrow V_{h}(O)$ be the Clement interpolant and define $\pi_{h, O}$ : $H^{s}(O) \rightarrow V_{h}(O)$ such that

$$
\pi_{h, O} v=\pi_{h, O, C l} E_{O} v
$$

Finally, we let $\pi_{h}:\left(\oplus_{i=1}^{N_{0}} H^{s}\left(\Omega_{i}\right)\right) \oplus\left(\oplus_{i=1}^{N} H^{s}\left(\Omega_{i}\right)\right) \rightarrow V_{h}$ be defined componentwise by $\left(\pi_{h} v\right)_{O}=\pi_{h, O} v_{O}$. We have the interpolation error estimates

$$
\left\|v-\pi_{h} v\right\|_{H^{m}(O)} \lesssim h^{s-m}\|v\|_{H^{s}(O)} \quad m=0,1
$$

for $0 \leq m \leq s \leq p+1$. For the $d$ dimensional domains $O=\Omega_{i}, i=1, \ldots, N$, we derive (3.30) using a standard interpolation error bound for the Clement interpolant followed by the stability of the extension operator

$$
\begin{aligned}
\left\|v-\pi_{h} E v\right\|_{H^{m}\left(\Omega_{i}\right)} \leq & \left\|v-\pi_{h} E v\right\|_{H^{m}\left(\mathcal{T}_{h, i}\right)} \\
& \leq h^{p+1-m}\|E v\|_{H^{p+1}\left(\mathcal{T}_{h, i}\right)} \leq h^{p+1-m}\|v\|_{H^{p+1}\left(\Omega_{i}\right)}
\end{aligned}
$$

For the $d-1$ dimensional skeleton subdomains $\Omega_{0, i}, i=1, \ldots, N_{0}$, we instead employ the trace inequality

$$
\|v\|_{\Omega_{0, i}}^{2} \lesssim h^{-1}\|v\|_{\mathcal{T}_{h, 0, i}}^{2}+h\|\nabla v\|_{\mathcal{T}_{h, 0, i}}^{2}
$$

and then 3.30 can be derived in the same way as in 3.31)-3.32).

Interpolation Error Estimate. The following estimate holds

$$
\left\|v-\pi_{h} v \mid\right\|_{h}^{2} \lesssim h^{2 p}\left\|v_{0}\right\|_{H^{p+1 / 2}\left(\Omega_{0}\right)}^{2}+\sum_{i=0}^{N} h^{2 p}\left\|v_{i}\right\|_{H^{p+1}\left(\Omega_{i}\right)}^{2}
$$

Remark 3.1. Note that here we can easily localize $h$ and $p$ to the subdomains and skeleton subdomains. But for clarity we keep a global mesh parameter $h$ and order of polynomials $p$. 
Proof of (3.34). Let $\eta=v-\pi_{h} v$ be the interpolation error and using the definition (3.1) of the energy norm we have

$$
\begin{aligned}
\|\eta\| \|_{h}^{2} & =\sum_{i=1}^{N}\|\nabla \eta\|_{\Omega_{i}}^{2}+h\|\nabla \eta\|_{\partial \Omega_{i}}^{2}+h^{-1}\left\|[\eta]_{i}\right\|_{\partial \Omega_{i}}^{2} \\
& \leq \sum_{i=1}^{N}\|\nabla \eta\|_{\Omega_{i}}^{2}+h\|\nabla \eta\|_{\partial \Omega_{i}}^{2}+h^{-1}\left\|\eta_{i}\right\|_{\partial \Omega_{i}}^{2}+h^{-1}\left\|\eta_{0}\right\|_{\partial \Omega_{i}}^{2} \\
& \lesssim \sum_{i=1}^{N}\|\nabla \eta\|_{\Omega_{i}}^{2}+h\|\nabla \eta\|_{\partial \Omega_{i}}^{2}+h^{-1}\left\|\eta_{i}\right\|_{\partial \Omega_{i}}^{2}+\sum_{i=1}^{N_{0}} h^{-1}\left\|\eta_{0}\right\|_{\Omega_{i, 0}}^{2} \\
& \lesssim \sum_{i=1}^{N}\|\nabla \eta\|_{\Omega_{i}}^{2}+\left(\|\nabla \eta\|_{\mathcal{T}_{h, i}\left(\partial \Omega_{i}\right)}^{2}+h^{2}\left\|\nabla^{2} \eta\right\|_{\mathcal{T}_{h, i}\left(\partial \Omega_{i}\right)}^{2}\right) \\
& +\left(h^{-2}\|\eta\|_{\mathcal{T}_{h, i}\left(\partial \Omega_{i}\right)}^{2}+\|\nabla \eta\|_{\mathcal{T}_{h, i}\left(\partial \Omega_{i}\right)}^{2}\right)+\sum_{i=1}^{N_{0}} 2 h^{-1}\left\|\eta_{0}\right\|_{\Omega_{i, 0}}^{2} \\
& \lesssim \sum_{i=1}^{N} h^{2 p}\|v\|_{H^{p+1}\left(\Omega_{i}\right)}^{2}+\sum_{i=1}^{N_{0}} h^{2 p}\left\|v_{0, i}\right\|_{H^{p+1 / 2}\left(\Omega_{0, i}\right)}^{2}
\end{aligned}
$$

Here we used the trace inequality

$$
\|v\|_{\partial \Omega_{i}}^{2} \lesssim h^{-1}\|v\|_{\mathcal{T}_{h, i}\left(\partial \Omega_{i}\right)}^{2}+h\|\nabla v\|_{\mathcal{T}_{h, i}\left(\partial \Omega_{i}\right)}^{2}
$$

where $\mathcal{T}_{h, i}\left(\partial \Omega_{i}\right)$ is the set of all elements in $\mathcal{T}_{h, i}$ which intersect $\partial \Omega_{i}$, and the interpolation estimate 3.30 .

\subsection{Stabilization Forms}

Define the following stabilization forms:

- For $i=1, \ldots, N$ define the stabilization forms

$$
s_{h, i}(v, w)=\sum_{l=1}^{p} c_{d, l} h^{2 l-1}\left(\left[D_{n}^{l} v\right],\left[D_{n}^{l} w\right]\right)_{\mathcal{F}_{h, i}}
$$

where $c_{d, l}>0$ is a parameter and $\mathcal{F}_{h, i}$ is the set of interior faces in $\mathcal{T}_{h}$ which belongs to an element that intersect the boundary $\partial \Omega_{i}$ and $D_{\xi}^{l} v$ denotes the contraction of $\xi^{l}$ and $\nabla^{l} v$.

- For $i=0$ recall that $\Omega_{0}=\cup_{j=0}^{N_{0}} \Omega_{0, j}$ and define

$$
s_{h, 0}=\sum_{j=1}^{N_{0}} s_{h, 0, j}
$$

where

$$
s_{h, 0, j}(v, w)=\sum_{l=1}^{p} c_{d-1, l} h^{2 l}\left(\left(D_{\nu}^{l} v, D_{\nu}^{l} w\right)_{\Omega_{0, j}}+\left(\left[D_{n}^{l} v\right],\left[D_{n}^{l} w\right]\right)_{\mathcal{F}_{h, 0, j}}\right)
$$

where $c_{d-1, l}>0$ is a parameter, $\nu$ is the normal to the smooth $d-1$ manifold $\Omega_{0, j}$ and $\mathcal{F}_{h, 0, j}$ denotes the set of interior faces in $\mathcal{T}_{h, 0, j}$.

Then the required properties of the stabilization forms $(2.17)-(2.20)$ hold, see [25, 28, 30]. 


\subsection{Error Estimates}

Energy Norm Error Estimate. The following estimate holds

$$
\left\|u-u_{h} \mid\right\|_{h}^{2} \lesssim h^{2 p}\left\|u_{0}\right\|_{H^{p+1 / 2}\left(\Omega_{0}\right)}^{2}+\sum_{i=0}^{N} h^{2 p}\left\|u_{i}\right\|_{H^{p+1}\left(\Omega_{i}\right)}^{2}
$$

This result follows immediately from the coercivity, Galerkin orthogonality, continuity, and interpolation error estimate. For the readers convenience we detail the proof.

Proof. Let $e_{h}=\pi_{h} u-u_{h}$ and $\rho=u-\pi_{h} u$. Then we have

$$
\begin{aligned}
\left\|e_{h}\right\|_{h}^{2} & \lesssim A_{h}\left(e_{h}, e_{h}\right) \\
& =A_{h}\left(\rho, e_{h}\right) \\
& \leq\|\rho\|\left\|_{h}\right\| e_{h}\|\|_{h} \\
& \left.\lesssim\left(h^{2 p}\left\|u_{0}\right\|_{H^{p+1 / 2}\left(\Omega_{0}\right)}^{2}+\sum_{i=0}^{N} h^{2 p}\left\|u_{i}\right\|_{H^{p+1}\left(\Omega_{i}\right)}^{2}\right)^{1 / 2}\left\|e_{h}\right\|\right|_{h}
\end{aligned}
$$

Subdomain $\boldsymbol{L}^{2}$ Error Estimates. Assuming that the solution to the dual problem

$$
-\nabla \cdot a \nabla \phi=\psi \text { in } \Omega, \quad \phi=0 \text { on } \partial \Omega
$$

has the regularity

$$
\sum_{i=1}^{N}\left\|\phi_{i}\right\|_{H^{s}\left(\Omega_{i}\right)}^{2} \lesssim\|\psi\|_{\Omega}^{2}, \quad s \in(3 / 2,1]
$$

we have the following error estimate

$$
\sum_{i=1}^{N}\left\|u_{i}-u_{h, i}\right\|_{\Omega_{i}}^{2} \lesssim h^{2 p+2 s}\left\|u_{0}\right\|_{H^{p+1 / 2}\left(\Omega_{0}\right)}^{2}+\sum_{i=0}^{N} h^{2 p+2 s}\left\|u_{i}\right\|_{H^{p+1}\left(\Omega_{i}\right)}^{2}
$$

Skeleton $\boldsymbol{L}^{\mathbf{2}}$ Error Estimates. In order to estimate the $L^{2}$ norm of the skeleton error we instead consider the dual problem

$$
\begin{aligned}
-\nabla \cdot a_{i} \nabla \phi_{i} & =0 & & \text { in } \Omega_{i} \\
\llbracket n \cdot a \nabla \phi \rrbracket & =\psi & & \text { on } \Omega_{0} \\
{[\phi]_{i} } & =0 & & \text { on } \partial \Omega_{i} \cap \Omega_{0} \\
\phi_{i} & =0 & & \text { on } \partial \Omega_{i} \cap \partial \Omega
\end{aligned}
$$

since $\psi$ is in $L^{2}$ on the skeleton the maximal regularity is

$$
\sum_{i=1}^{N}\left\|\phi_{i}\right\|_{H^{3 / 2}\left(\Omega_{i}\right)}^{2} \lesssim\|\psi\|_{\Omega_{0}}^{2}
$$

and thus we arrive at the estimate

$$
\left\|u_{0}-u_{h, 0}\right\|^{2} \lesssim h^{2 p+1}\left\|u_{0}\right\|_{H^{p+1 / 2}\left(\Omega_{0}\right)}^{2}+\sum_{i=0}^{N} h^{2 p+1}\left\|u_{i}\right\|_{H^{p+1}\left(\Omega_{i}\right)}^{2}
$$

Observe that this shows that the error in the discrete $H^{1 / 2}$ norm, $h^{-\frac{1}{2}}\left\|u_{0}-u_{h, 0}\right\|$ has similar convergence order as the energy norm error, which is optimal. 


\section{The Schur Complement}

In this section we show how the bulk fields can be eliminated using the Schur complement and we derive a bound for the condition number of stiffness matrix associated with the Schur complement.

\subsection{Definitions}

- Define the operator $T_{h}: V_{h, 0} \rightarrow V_{h, 1, N}=\bigoplus_{i=1}^{N} V_{h, i}$ such that

$$
A_{h}\left(v_{0}+T_{h} v_{0}, w\right)=0 \quad \forall w \in V_{h, 1, N}
$$

- Define the Schur complement form on $V_{h, 0}$ by

$$
S_{h}(v, w)_{\Omega_{0}}=A_{h}\left(v+T_{h} v, w+T_{h} w\right) \quad v, w \in V_{h, 0}
$$

- Define the energy norm on $V_{h, 0}$ associated with the Schur complement by

$$
\left\|v_{0}\right\|_{S_{h}}=\left\||| v_{0}+T_{h} v_{0}\right\|_{h}
$$

- It follows directly from the definition of the Schur complement form that $S_{h}$ is coercive and continuous on $V_{h, 0}$.

\subsection{Solution Using The Schur Complement}

Note that we have the $A_{h}$-orthogonal splitting

$$
W_{h}=\left(I+T_{h}\right) V_{h, 0} \perp\left(\{0\} \oplus V_{h, 1, N}\right)
$$

and thus $u_{h}=\left(I+T_{h}\right) u_{h, 0}+\left(0 \oplus u_{h, 1, N}\right)$ where $u_{h, 0} \in V_{h, 0}$ is the solution to

$$
S_{h}\left(u_{h, 0}, v_{0}\right)=l_{h}\left(\left(I+T_{h}\right) v_{0}\right) \quad \forall v_{0} \in V_{h, 0}
$$

and $u_{h, 1, N}$ is the solution to

$$
A_{h}\left(0 \oplus u_{h, 1, N}, 0 \oplus v\right)=l_{h}(v) \quad \forall v \in V_{h, 1, N}
$$

where we used the notation $0 \oplus u_{h, 1, N}$ to indicate that the component in $V_{h, 0}$ is zero. We note that (4.6) decouples and can be solved subdomain wise. For each subdomain we get a Nitsche type formulation with homogeneous Dirichlet data and right hand side $f_{i}=\left.f\right|_{\Omega_{i}}$.

\subsection{Some Basic Estimates}

We collect the basic bounds needed to show an estimate of the condition number of the stiffness matrix associated with the Schur complement.

Norm Equivalence. There are constants such that

$$
\inf _{w \in V_{h, 1, N}}\left\|\left|v_{0}+w\right|\right\|_{h} \lesssim\left\|\left|\left\|v_{0}\right\|\right|_{S_{h}} \lesssim \inf _{w \in V_{h, 1, N}}\right\| \mid v_{0}+w \|_{h}
$$

Proof of (4.7). 1. By definition

$$
\inf _{w \in V_{h, 1, N}}\left\|\left|v_{0}+w\right|\right\|_{h} \lesssim\left\||| v_{0}+T_{h} v_{0}\right\|_{h}=\|\| v_{0} \|\left.\right|_{S_{h}}
$$


2. We have

$$
\begin{aligned}
\left\|\left|v_{0} \|\right|_{S_{h}}^{2}\right. & =\left.\left\|v_{0}+T_{h} v_{0}\right\|\right|_{h} ^{2} \\
& \lesssim A_{h}\left(v_{0}+T_{h} v_{0}, v_{0}+T_{h} v_{0}\right) \\
& =A_{h}\left(v_{0}+T_{h} v_{0}, v_{0}+w\right) \\
& \lesssim\left\|v_{0}+T_{h} v_{0}\right\|\left\|_{h}\right\| \mid v_{0}+w \|_{h}
\end{aligned}
$$

where we used the coercivity (3.3) of $A_{h}$, the orthogonality 4.1$)$ of $T_{h}$, and the continuity (3.2) of $A_{h}$. Thus we conclude that

$$
\left\|\mid v_{0}\right\|_{h} \lesssim\left\|v_{0}+w\right\|_{h}
$$

for all $w \in V_{h, 1, N}$, and therefore

$$
\left\|v_{0}+T_{h} v_{0} \mid\right\|\left\|_{h} \inf _{w \in V_{h, 1, N}}\right\| v_{0}+w \|_{h}
$$

Skeleton Poincaré Estimate. There is a constant such that for all $v \in V_{h, 0}$,

$$
\left(\min _{1 \leq i \leq N} d_{\Omega_{i}}\right) h^{-1}\|v\|_{\mathcal{T}_{h, 0}}^{2} \lesssim\|v\|_{S_{h}}^{2}
$$

Proof of (4.15). Using the Poincaré inequality (3.27) we have for any $w \in V_{h, 1, N}$,

$$
\left(\min _{1 \leq i \leq N} d_{\Omega_{i}}\right) h^{-1}\|v\|_{\mathcal{T}_{h, 0}}^{2} \lesssim\|\| v+w \|_{h}^{2}
$$

and as a consequence

$$
\left(\min _{1 \leq i \leq N} d_{\Omega_{i}}\right) h^{-1}\|v\|_{\mathcal{T}_{h, 0}}^{2} \lesssim\left\|v+T_{h} v\right\|_{h}^{2}=\|\| v \|_{S_{h}}^{2}
$$

Skeleton Inverse Estimate. There is a constant such that for all $v \in V_{h, 0}$,

$$
\|v \mid\|_{S_{h}}^{2} \lesssim h^{-1}\left(\|v\|_{\Omega_{0}}^{2}+\|v\|_{s_{h, 0}}^{2}\right)
$$

Proof of (4.18). We have

$$
\begin{aligned}
\left\|v_{0} \mid\right\|_{S_{h}}^{2} & \lesssim \inf _{w \in V_{h, 1, N}}\left\|v_{0}+w \mid\right\|_{h}^{2} \\
& \lesssim\left\|\left|v_{0}+0\right|\right\|_{h}^{2} \\
& =\sum_{i=1}^{N} h^{-1}\left\|v_{0}\right\|_{\partial \Omega_{i}}^{2}+h^{-1}\|v\|_{s_{h, 0}}^{2} \\
& \lesssim h^{-1}\left(\|v\|_{\Omega_{0}}^{2}+\|v\|_{s_{h, 0}}^{2}\right)
\end{aligned}
$$

where we choose $w=0$ on $V_{h, 1, N}$ and then used the definition of the energy norm. 


\subsection{Condition Number Estimate for the Schur Complement Definitions and Basic Results.}

- Let $\left\{\varphi_{i}\right\}_{i=1}^{D}$ be the basis in $V_{h, 0}$ and denote the expansion by

$$
v=\sum_{i=1}^{D} \widehat{v}_{i} \varphi_{i}
$$

Then we have the equivalence

$$
\|v\|_{T_{h, 0}}^{2} \sim h^{d}\|\widehat{v}\|_{\mathbb{R}^{D}}^{2}
$$

- The stiffness matrix associated with the Schur complement is defined by

$$
(\widehat{S} \widehat{v}, \widehat{w})_{\mathbb{R}^{D}}=S_{h}(v, w)
$$

Then $\widehat{S}$ is SPD and thus the spectrum is real and positive, and we have the Rayleigh quotient characterization of the largest and smallest eigenvalues

$$
\lambda_{\max }=\max _{\hat{v} \in \mathbb{R}^{D} \backslash\{0\}} \frac{(\widehat{S} \widehat{v}, \widehat{v})_{\mathbb{R}^{D}}}{\|\widehat{v}\|_{\mathbb{R}^{D}}^{2}}, \quad \lambda_{\min }=\min _{\hat{v} \in \mathbb{R}^{D} \backslash\{0\}} \frac{(\widehat{S} \widehat{v}, \widehat{v})_{\mathbb{R}^{D}}}{\|\widehat{v}\|_{\mathbb{R}^{D}}^{2}}
$$

- The condition number is defined by

$$
\kappa=\frac{\lambda_{\max }}{\lambda_{\min }}
$$

where $\lambda_{\max }$ and $\lambda_{\min }$ denote the maximum and minimum eigenvalues of $\widehat{S}$. In view of (4.26) we have the identity

$$
\kappa=\max _{\widehat{v} \in \mathbb{R}^{D} \backslash\{0\}} \frac{(\widehat{S} \widehat{v}, \widehat{v})_{\mathbb{R}^{D}}}{\|\widehat{v}\|_{\mathbb{R}^{D}}^{2}} \max _{\hat{v} \in \mathbb{R}^{D} \backslash\{0\}} \frac{\|\widehat{v}\|_{\mathbb{R}^{D}}^{2}}{(\widehat{S} \widehat{v}, \widehat{v})_{\mathbb{R}^{D}}}
$$

Condition Number Estimate. The condition number satisfies the estimate

$$
\kappa(\widehat{S}) \lesssim h^{-1}\left(\min _{1 \leq i \leq N} d_{\Omega_{i}}\right)^{-1}
$$

Proof. The bound follows directly from the following two estimates:

1. We have

$$
\begin{aligned}
(\widehat{S} \widehat{v}, \widehat{v})_{\mathbb{R}^{D}} & =A_{h}\left(v+T_{h} v, v+T_{h} v\right) \\
& \lesssim\left\|v+T_{h} v\right\|_{h}^{2} \\
& \lesssim h^{-1}\left(\|v\|_{L^{2}\left(\Omega_{0}\right)}^{2}+\|v\|_{s_{h, 0}}^{2}\right) \\
& \lesssim h^{-2}\|v\|_{\mathcal{T}_{h, 0}}^{2} \\
& \lesssim h^{-2} h^{d}\|\widehat{v}\|_{\mathbb{R}^{D}}^{2}
\end{aligned}
$$

where we used the continuity (3.2) of $A_{h}$, the inverse inequality (4.18), and the equivalences (4.24) and (2.18). Thus we conclude that

$$
\lambda_{\max } \lesssim h^{d-2}
$$


2. We have

$$
\begin{aligned}
(\widehat{S} \widehat{v}, \widehat{v})_{\mathbb{R}^{D}} & =A_{h}\left(v+T_{h} v, v+T_{h} v\right) \\
& \gtrsim\left\|v+T_{h} v\right\|_{h}^{2} \\
& \gtrsim\left(\min _{1 \leq i \leq N} d_{\Omega_{i}}\right) h^{-1}\|v\|_{L^{2}\left(\mathcal{T}_{h, 0}\right)}^{2} \\
& \gtrsim\left(\min _{1 \leq i \leq N} d_{\Omega_{i}}\right) h^{-1} h^{d}\|\widehat{v}\|_{\mathbb{R}^{D}}^{2}
\end{aligned}
$$

where we used the coercivity (3.3) of $A_{h}$, the Poincaré estimate (4.15), and the equivalence (4.24). We conclude that

$$
\lambda_{\min }^{-1} \lesssim h^{-(d-1)}\left(\min _{1 \leq i \leq N} d_{\Omega_{i}}\right)^{-1}
$$

\section{$5 \quad$ Numerical Results}

For assessment and illustration we implemented a 2D version of the method. We first give some details on implementation choices and then we present some illustrating examples and convergence results.

\subsection{Implementation}

Approximation Spaces. On each subdomain and skeleton subdomain we define an approximation space by a mesh equipped with some finite elements. In all examples below we use standard Lagrange elements of degree $p$ which on quadrilaterals are tensor product polynomial elements $Q_{p}$ and on triangles are full polynomial elements $P_{p}$. While the mesh on each subdomain and skeleton subdomain could be constructed completely independent of each other we here focus on two cases:

- Global Background Grid. All meshes, i.e. subdomain meshes $\left\{\mathcal{T}_{h, i}\right\}_{i=1}^{N}$ and skeleton subdomain meshes $\left\{\mathcal{T}_{h, 0, k}\right\}_{k=1}^{N_{0}}$, are extracted from the same background grid $\mathcal{T}_{h, \Omega}$.

- Single Element Interfaces. Subdomain meshes $\left\{\mathcal{T}_{h, i}\right\}_{i=1}^{N}$ may be arbitrarily constructed while each skeleton subdomain $\Omega_{0, k}, 1 \leq k \leq N_{0}$, is equipped with a mesh $\mathcal{T}_{h, 0, k}$ consisting of a single, typically higher order, element in $\mathbb{R}^{d}$.

The benefit of either choice is that the implementation of the Nitsche terms $(2.30)-(2.31)$ becomes particularly straightforward as we for $T \in \mathcal{T}_{h, i}, \partial \Omega_{i} \cap T \neq \emptyset$, trivially know the element of the neighboring skeleton subdomain mesh and that $\partial \Omega_{i} \cap T$ is completely contained within that element. More general cases require the construction of the union of subdomain meshes and skeleton subdomain meshes to correctly evaluate the skeleton integrals. For curved skeletons, parametrically mapped subdomains or simply the case $d=3$ this construction is typically challenging to perform in a robust and efficient manner.

Parameter Choices. For the Nitsche penalty parameter we choose $\beta=10 \cdot p^{2}$ and for the stabilization parameters we, as in the numerical examples in [28], choose $c_{d, l}=c_{d-1, l}=$ $\frac{10^{-3}}{l !}$. We note no particular sensitivity in the choice of values for these parameters. 


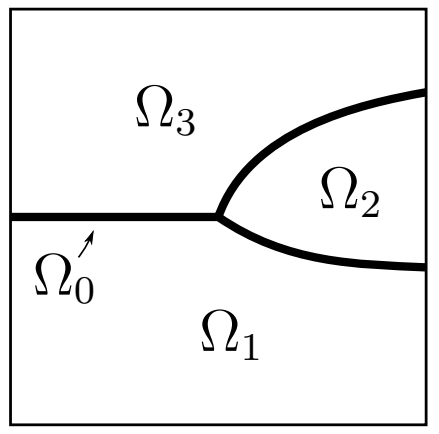

Figure 2: Illustrations of the model problem geometry. The unit square $[0,1]^{2}$ is divided into three subdomains according to the figure with material coefficients $a_{1}=1, a_{2}=2$ and $a_{3}=3$.

\section{$5.2 \quad$ Numerical Examples}

For our numerical examples we consider different partitions of the unit square $[0,1]^{2}$. We let the right hand side be given by $f=1$ and we vary the material coefficient $a$ in each subdomain.

Example 1: Three Subdomains. For our first numerical example we partition the unit square into three subdomains with a different constant material coefficient in each subdomain, see Figure 2. Thus, we in this problem have three skeleton subdomains. We consider both of the mesh constructions described in Section 5.1 .

- Global Background Grid. Here all meshes are extracted from the same background grid, see Figure 3, and we use $Q_{2}$ elements on each mesh. Note that all subdomains have cut elements and that some skeleton subdomains are curved within elements. In this setting there are no locking effects due to non-matching approximation spaces when choosing the penalty parameter $\beta$ large. A sample solution and the magnitude of its gradient are presented in Figure 4 .

- Single Element Interfaces. Here the mesh on each subdomain is constructed independently, some as quadrilateral meshes and some as triangular, and we equip all subdomain meshes with $Q_{2} / P_{2}$ elements. On each skeleton subdomain we use a single $Q_{4}$ element. Sample meshes in this set-up are visualized in Figure 5 and the corresponding numerical solution is presented in Figure 6.

Example 2: Voronoi Diagram. In our second example we construct a subdivision of the unit square by generating a Voronoi diagram from 50 uniformly distributed random points in $[0,1]^{2}$ and taking the restriction of this diagram to the unit square, see Figure 7 . We again consider the two different set-ups regarding mesh construction albeit we make different choices for the material coefficient in the two cases.

- Global Background Grid. Here we extract all meshes from the same background grid and we equip our meshes with Lagrange $Q_{2}$ elements. The material coefficient is constant on each subdomain $\Omega_{i}$ and is chosen as $a_{i}=0.01+X$ where $X \in[0,1]$ is a uniformly distributed random variable. This set-up is illustrated in Figure 7a. We can easily generate background grids of any mesh size and in Figure 8 we present results 


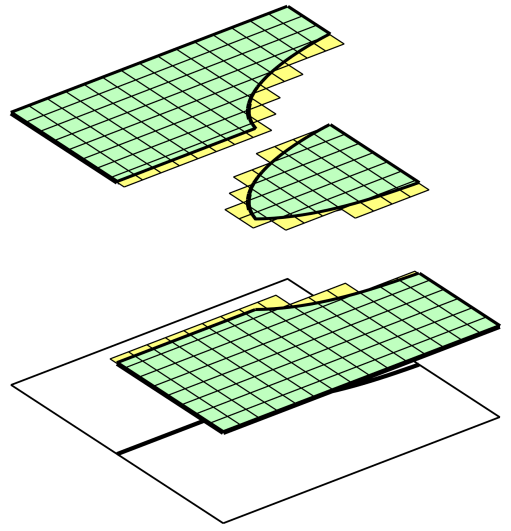

(a) Subdomain meshes

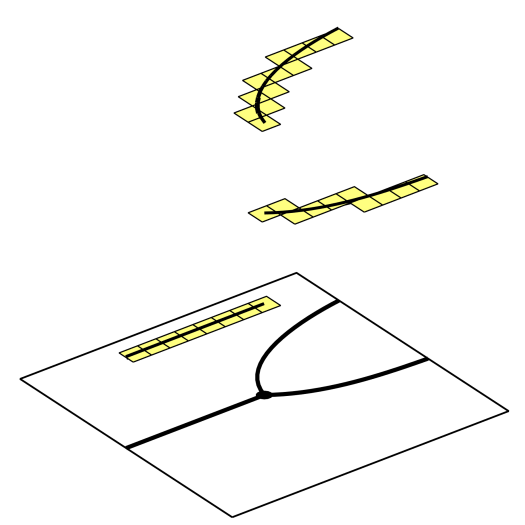

(b) Skeleton subdomain meshes

Figure 3: Meshes in the three subdomains example extracted from a global background grid. (a) Subdomain solutions are approximated using $Q_{2}$ elements. (b) Skeleton subdomain solutions are approximated in an embedding space of $Q_{2}$ elements.

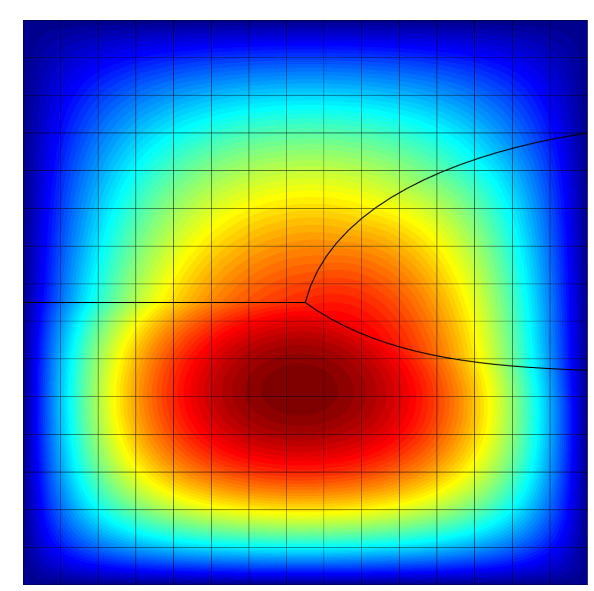

(a) Solution

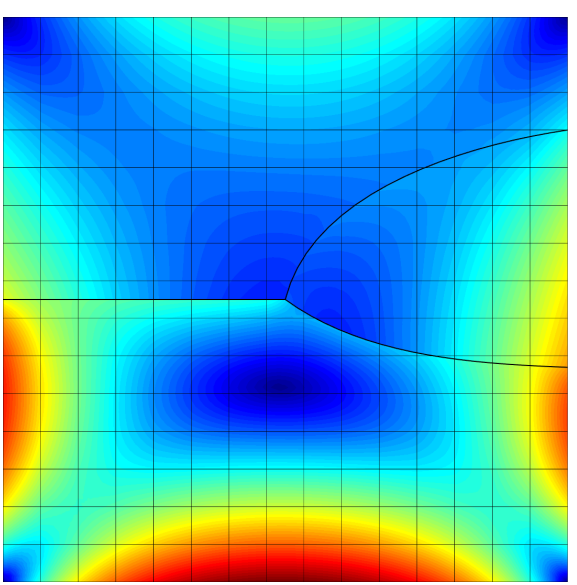

(b) Gradient magnitude

Figure 4: Three subdomains with different material coefficients and $Q_{2}$ meshes extracted from a global background grid. 


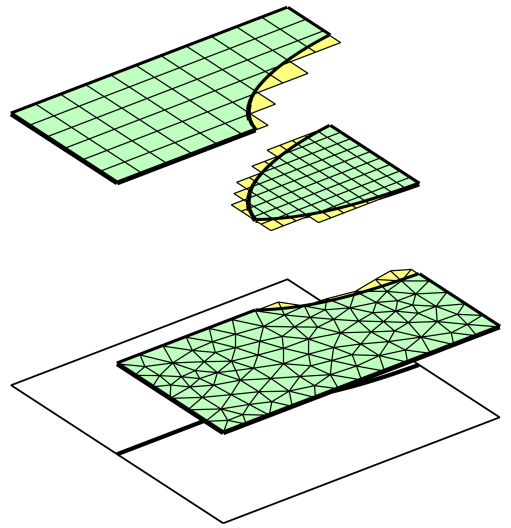

(a) Subdomain meshes

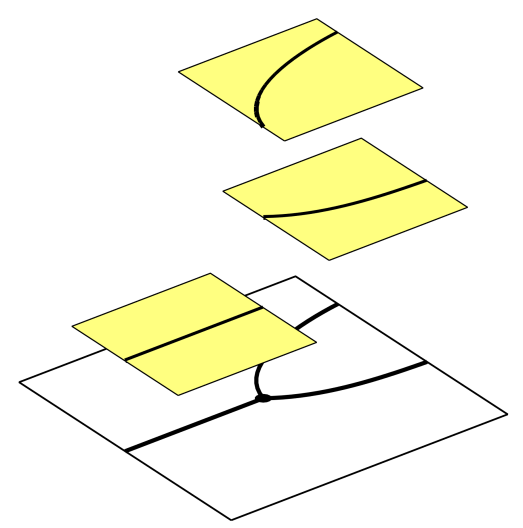

(b) Skeleton subdomain meshes

Figure 5: Meshes in the three subdomains example with skeleton subdomains embedded in a single element. (a) Subdomain solutions are approximated using $Q_{2}$ and $P_{2}$ elements on quadrilateral respectively triangle meshes. (b) Each skeleton subdomain is embedded in a single $Q_{4}$ element.

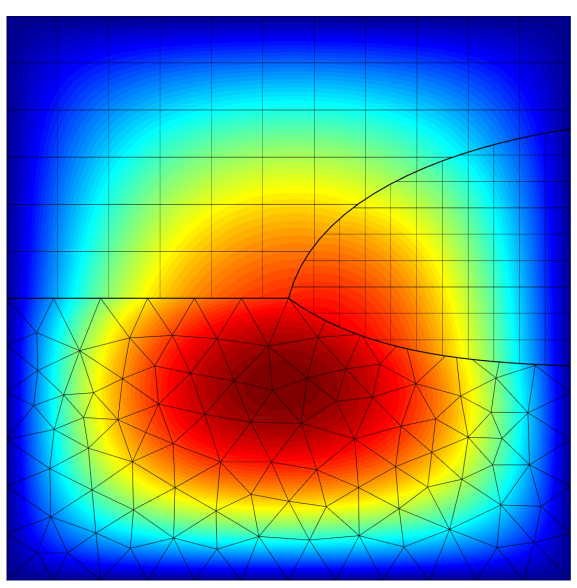

(a) Solution

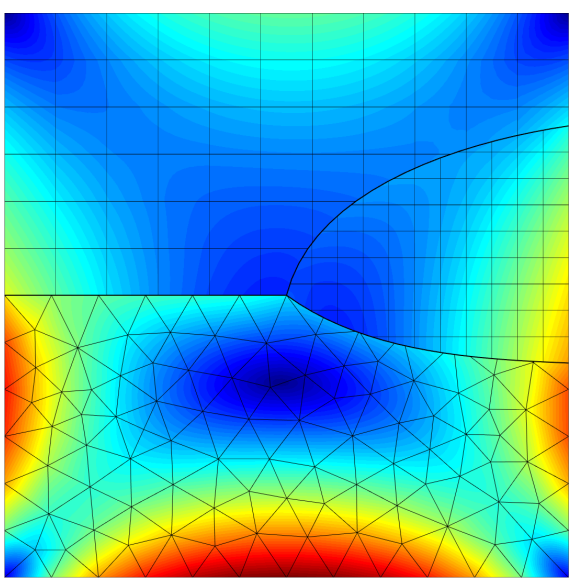

(b) Gradient magnitude

Figure 6: Three subdomains with different material coefficients and nonmatching meshes. Here meshes with $Q_{2} / P_{2}$ elements are used on each subdomain. On each skeleton subdomain the solution is approximated using a single $Q_{4}$ element. 


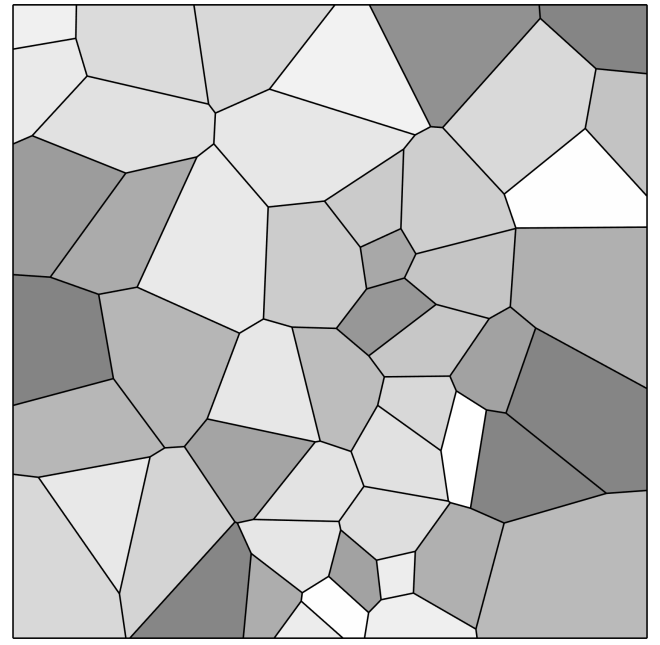

(a) Subdomain-wise coefficient $a$

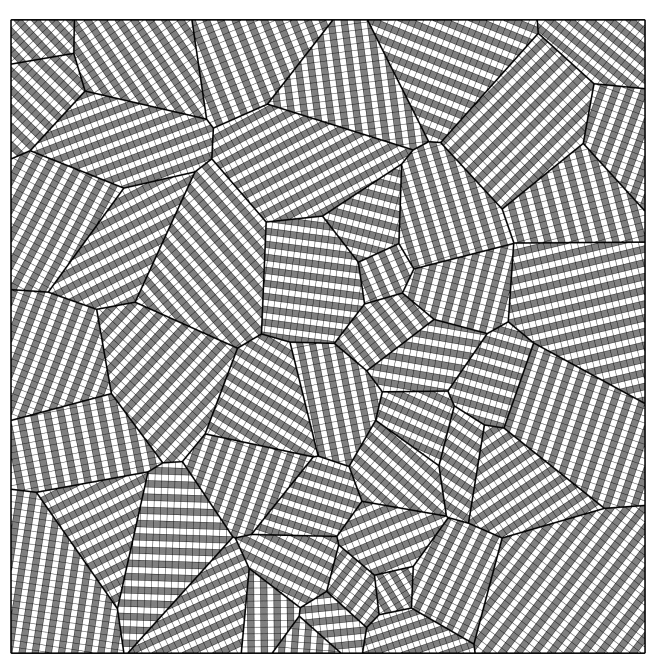

(b) Element-wise coefficient $a$

Figure 7: Subdivisions of the unit square $[0,1]^{2}$ generated from Voronoi diagrams featuring varying material coefficients. (a) Domain with material coefficient $a \in[0.01,1]$ which is constant within each subdomain and chosen using a uniformly distributed random variable. (b) Domain with a randomly oriented mesh in each subdomain and a material coefficient $a$ which alternates between 1 and 1000 row-wise in the mesh.

for three different mesh sizes. In Figures $8 \mathrm{c} 8 \mathrm{~d}$ we note that the method also works well when the mesh size is of the same order as the subdomain sizes. The extreme case where we only have a single element on each subdomain is presented in Figures $8 \mathrm{e} 8 \mathrm{f}$. This is much like a hybridized version of so called polygonal/polyhedral elements, see the overview in [13]. Note that in this extreme case we construct the single elements such that they are as small as possible while still containing its subdomain to avoid conditioning problems.

- Single Element Interfaces. In the situation where we equip skeleton subdomain with a single Lagrange $Q_{4}$ element we choose another set-up regarding meshes and material coefficient in each bulk subdomain. We randomly orient a fine mesh equipped with Lagrange $Q_{2}$ elements on each subdomain and we let the material coefficient alternate between 1 and 1000 row-wise in the mesh. This set-up is illustrated in Figure 7b and the numerical solution is presented in Figure 9. Note that this case is mainly an illustration of how we conveniently can implement cases where the subdomains are defined via mappings, in this case a rotation. Of course we here loose fine scale information across the skeleton and we have made no special adaption to handle the large variation in the material coefficient.

\subsection{Convergence Studies}

To study the convergence of the method in energy and $L 2$ norms we partition the unit square $\Omega$ into its left and right halves, $\Omega_{1}$ and $\Omega_{2}$, and we manufacture a problem with known exact solution from the ansatz

$$
u=\left\{u_{0}=\frac{1}{2} \sin (\pi y), u_{1}=x \sin (\pi y), u_{2}=(1-x-\sin (2 \pi x)) \sin (\pi y)\right\}
$$




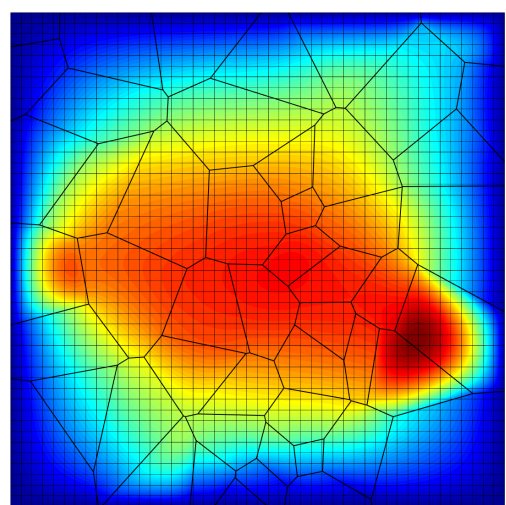

(a) $u_{h}, h=2^{-5}$

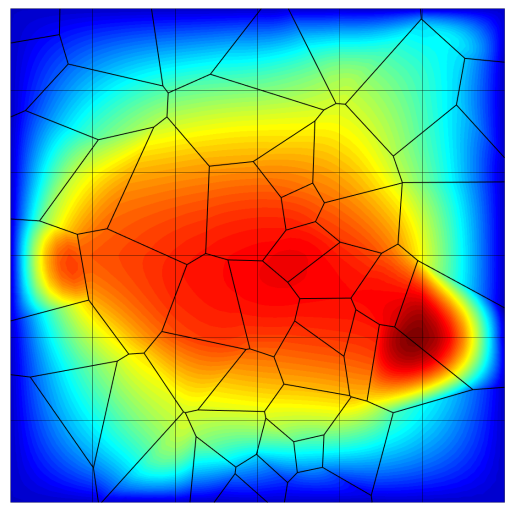

(c) $u_{h}, h=2^{-2}$

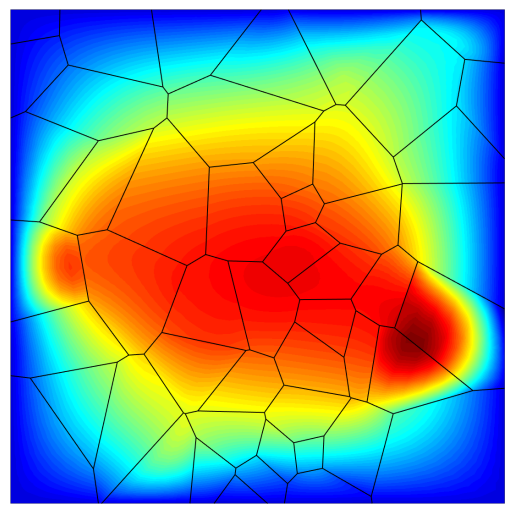

(e) $u_{h}, h=1$

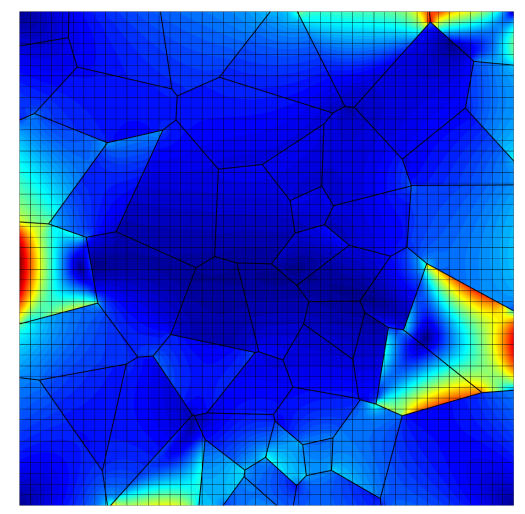

(b) $\left|\nabla u_{h}\right|, h=2^{-5}$

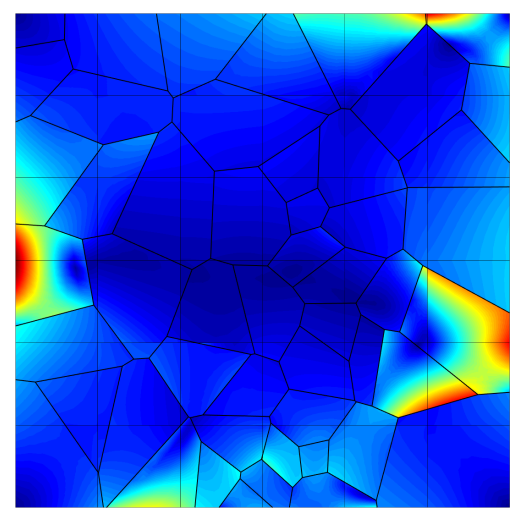

(d) $\left|\nabla u_{h}\right|, h=2^{-2}$

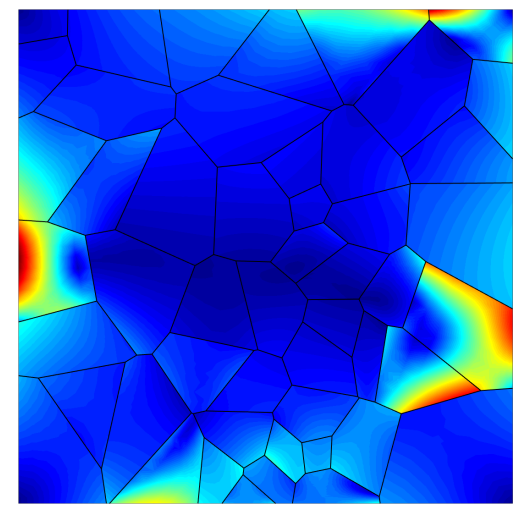

(f) $\left|\nabla u_{h}\right|, h=1$

Figure 8: Numerical solution $u_{h}$ and gradient magnitude $\left|\nabla u_{h}\right|$ on a Voronoi diagram subdivision with subdomain-wise constant material coefficient, see Figure 7a. (a)-(b) $Q_{2}$ elements on meshes generated from one fine grid. (c)(d) $Q_{2}$ elements on meshes generated from one coarse grid with a mesh size in the same order as the subdomain sizes. (e)-(f) A single $Q_{2}$ element on each subdomain and skeleton subdomain. 


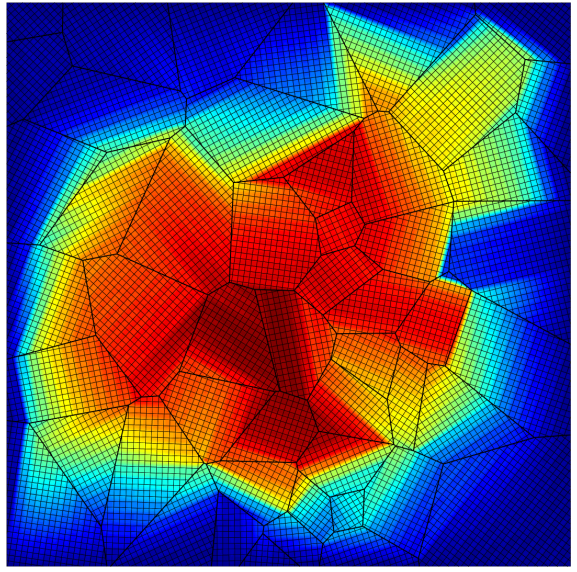

(a) Solution

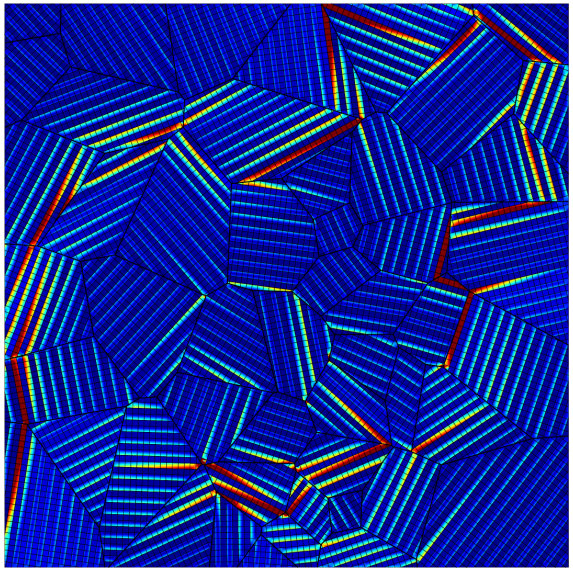

(b) Gradient magnitude

Figure 9: Numerical solution $u_{h}$ and gradient magnitude $\left|\nabla u_{h}\right|$ on a Voronoi diagram subdivision with a fine scale material coefficient pattern. On each subdomain a randomly oriented $Q_{2}$ mesh with a row-wise alternating material coefficient is set-up, see Figure 7b. The numerical solution on each skeleton subdomain is instead approximated by a single $Q_{4}$ element.

with coefficients $a_{1}=(2 \pi-1), a_{2}=1$. The domain, exact solution and exact gradient magnitude for this problem are displayed in Figure 10 .

- Global Background Grid. In Figure 11 we present convergence results in the case where all meshes are extracted form the same quadrilateral background grid using $Q_{p}$ elements, $p=1,2,3$, and we achieve the expected convergence rates of $O\left(h^{p}\right)$ and $O\left(h^{p+1}\right)$ in the energy and the $L^{2}$ norm, respectively.

- Single Element Interfaces. In this case we instead use $Q_{2}$ elements of size $h$ on each subdomain but only a single Lagrange $Q_{p}$ element, $p=2,4,6$, on each skeleton subdomain. As the skeleton subdomain meshes are not refined with smaller $h$ this naturally imposes a lower bound on the errors, which we also note in the convergence results presented in Figure 12

Acknowledgements. This research was supported in part by the Swedish Foundation for Strategic Research Grant No. AM13-0029, the Swedish Research Council Grants Nos. 2013-4708, 2017-03911, and the Swedish Research Programme Essence. EB was supported by EPSRC research grants EP/P01576X/1 and EP/P012434/1.

\section{References}

[1] L. Beirão da Veiga, F. Brezzi, L. D. Marini, and A. Russo. The hitchhiker's guide to the virtual element method. Math. Models Methods Appl. Sci., 24(8):1541-1573, 2014.

[2] S. P. A. Bordas, E. Burman, M. G. Larson, and M. Olshanskii, editors. Geometrically Unfitted Finite Element Methods and Applications, volume 121 of Lecture Notes in Computational Science and Engineering. Springer International Publishing, 2018.

[3] E. Burman. Ghost penalty. C. R. Math. Acad. Sci. Paris, 348(21-22):1217-1220, 2010. 


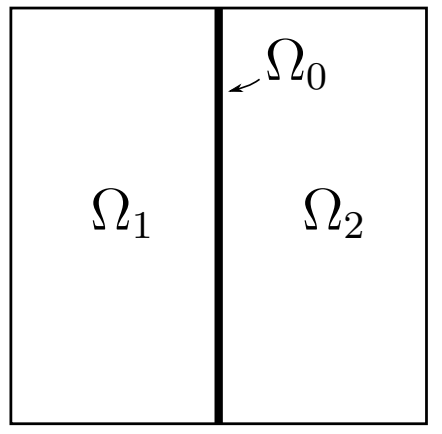

(a) Domain

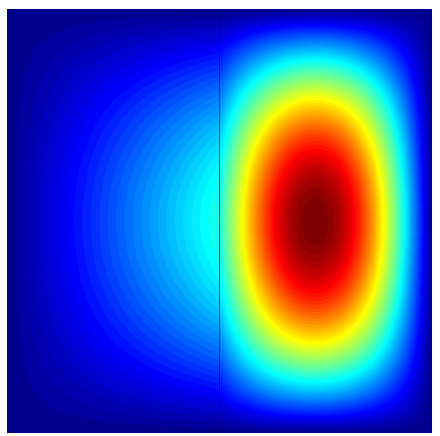

(b) Solution

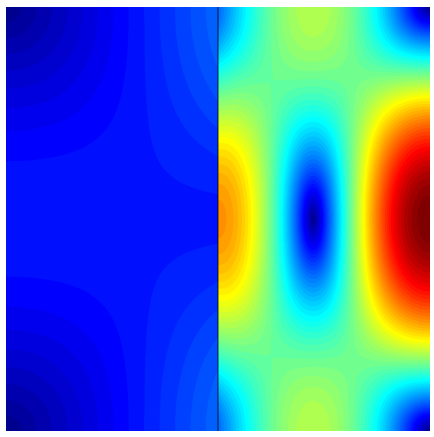

(c) Gradient magnitude

Figure 10: Problem with known exact solution used in convergence studies. (a) The domain is the unit square $[0,1]^{2}$ partitioned into two mirror symmetric subdomains according to the figure with material coefficients $a_{1}=1$ and $a_{2}=2 \pi-1$. (b) The exact solution (5.1). (c) The gradient magnitude of the exact solution.

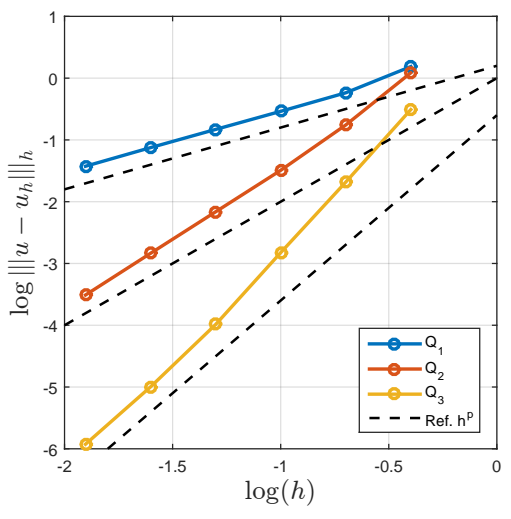

(a) Energy norm

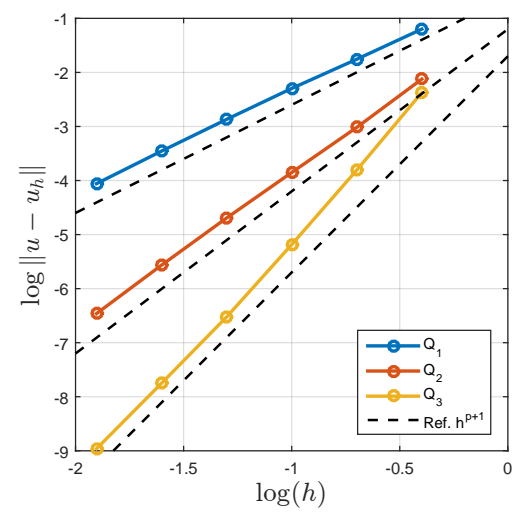

(b) $L^{2}$ norm

Figure 11: Convergence studies using meshes all from the same background grid. In all meshes the same elements are used $\left(Q_{1}-Q_{3}\right)$ and we achieve what we expect to be optimal convergence rates of $O\left(h^{p}\right)$ in the energy norm (a) respectively $O\left(h^{p+1}\right)$ in the $L^{2}$ norm (b). 


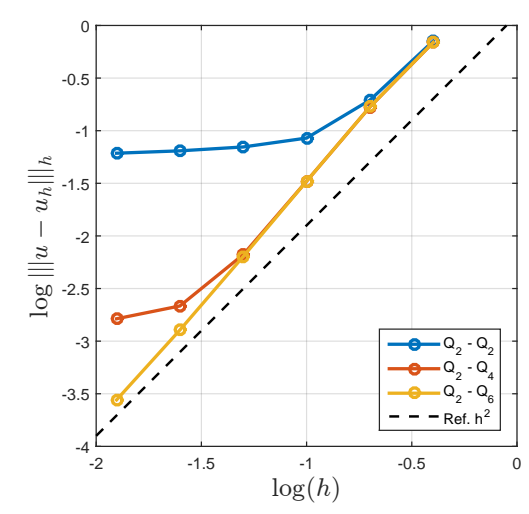

(a) Energy norm

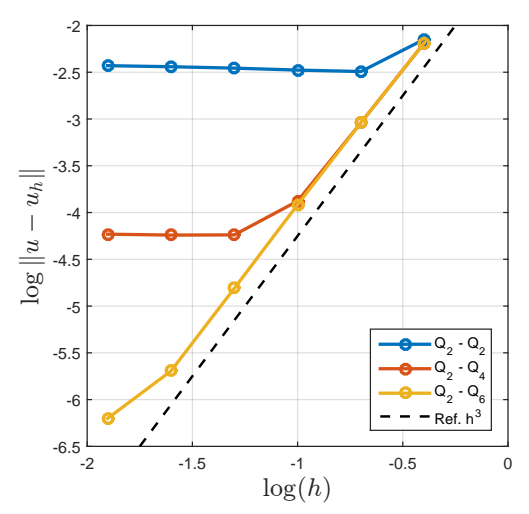

(b) $L^{2}$ norm

Figure 12: Convergence studies using non-matching meshes for the subdomains and a single polynomial for each skeleton subdomain. On the subdomains $Q_{2}$ elements are used and on the skeleton subdomains $Q_{2}-Q_{6}$ polynomials are used. Because there is no $h$ refinement of the skeleton subdomains the convergence levels out as the polynomial approximations on the skeleton become the dominant source of error.

[4] E. Burman, S. Claus, P. Hansbo, M. G. Larson, and A. Massing. CutFEM: discretizing geometry and partial differential equations. Internat. J. Numer. Meth. Engrg, 104(7):472-501, 2015.

[5] E. Burman, D. Elfverson, P. Hansbo, M. G. Larson, and K. Larsson. Shape optimization using the cut finite element method. Comput. Methods Appl. Mech. Engrg., 328:242-261, 2018.

[6] E. Burman and P. Hansbo. Fictitious domain finite element methods using cut elements: II. A stabilized Nitsche method. Appl. Numer. Math., 62(4):328-341, 2012.

[7] E. Burman, P. Hansbo, and M. G. Larson. A stabilized cut finite element method for partial differential equations on surfaces: the Laplace-Beltrami operator. Comput. Methods Appl. Mech. Engrg., 285:188-207, 2015.

[8] E. Burman, P. Hansbo, and M. G. Larson. A cut finite element method with boundary value correction. Math. Comp., 87(310):633-657, 2018.

[9] E. Burman, P. Hansbo, and M. G. Larson. Low regularity estimates for CutFEM approximations of an elliptic problem with mixed boundary conditions. Technical report, Mathematics, Umeå University, Sweden, 2018.

[10] E. Burman, P. Hansbo, M. G. Larson, and K. Larsson. Cut finite elements for convection in fractured domains. Comput. $\mathcal{E}$ Fluids (in press), 2018.

[11] E. Burman, P. Hansbo, M. G. Larson, and A. Massing. Cut finite element methods for partial differential equations on embedded manifolds of arbitrary codimensions. ESAIM Math. Model. Numer. Anal. (in press), Oct. 2018.

[12] A. Cangiani, Z. Dong, E. H. Georgoulis, and P. Houston. hp-version discontinuous Galerkin methods on polygonal and polyhedral meshes. SpringerBriefs in Mathematics. Springer, Cham, 2017.

[13] A. Cangiani, E. H. Georgoulis, and P. Houston. $h p$-version discontinuous Galerkin methods on polygonal and polyhedral meshes. Math. Models Methods Appl. Sci., 24(10):2009-2041, 2014.

[14] B. Cockburn, D. A. Di Pietro, and A. Ern. Bridging the hybrid high-order and hybridizable discontinuous Galerkin methods. ESAIM Math. Model. Numer. Anal., 50(3):635-650, 2016.

[15] B. Cockburn, J. Gopalakrishnan, and R. Lazarov. Unified hybridization of discontinuous Galerkin, mixed, and continuous Galerkin methods for second order elliptic problems. SIAM J. Numer. Anal., 47(2):1319-1365, 2009.

[16] D. A. Di Pietro, A. Ern, and S. Lemaire. An arbitrary-order and compact-stencil discretization of diffusion on general meshes based on local reconstruction operators. Comput. Methods Appl. Math., 14(4):461-472, 2014. 
[17] H. Egger. A class of hybrid mortar finite element methods for interface problems with non-matching meshes. Preprint: AICES-2009-2, January 2009.

[18] H. Egger and J. Schöberl. A hybrid mixed discontinuous Galerkin finite-element method for convection-diffusion problems. IMA J. Numer. Anal., 30(4):1206-1234, 2010.

[19] H. Egger and C. Waluga. A hybrid mortar method for incompressible flow. Int. J. Num. Anal. Mod., 9:793-812, 2012.

[20] H. Egger and C. Waluga. $h p$ analysis of a hybrid DG method for Stokes flow. IMA J. Numer. Anal., 33(2):687-721, 2013.

[21] J. Grande, C. Lehrenfeld, and A. Reusken. Analysis of a high-order trace finite element method for PDEs on level set surfaces. SIAM J. Numer. Anal., 56(1):228-255, 2018.

[22] A. Hansbo and P. Hansbo. An unfitted finite element method, based on Nitsche's method, for elliptic interface problems. Comput. Methods Appl. Mech. Engrg., 191(47-48):5537-5552, 2002.

[23] A. Hansbo, P. Hansbo, and M. G. Larson. A finite element method on composite grids based on Nitsche's method. ESAIM Math. Model. Numer. Anal., 37(3):495-514, 2003.

[24] P. Hansbo, T. Jonsson, M. G. Larson, and K. Larsson. A Nitsche method for elliptic problems on composite surfaces. Comput. Methods Appl. Mech. Engrg., 326:505-525, 2017.

[25] P. Hansbo, M. G. Larson, and K. Larsson. Cut finite element methods for linear elasticity problems. In S. Bordas, E. Burman, M. Larson, and M. Olshanskii, editors, Geometrically Unfitted Finite Element Methods and Applications, volume 121 of Lecture Notes in Comput. Sci. Eng. Springer, 2018.

[26] R. B. Kellogg. On the Poisson equation with intersecting interfaces. Applicable Anal., 4:101-129, 1974/75. Collection of articles dedicated to Nikolai Ivanovich Muskhelishvili.

[27] J. Könnö and R. Stenberg. Numerical computations with $H$ (div)-finite elements for the Brinkman problem. Comput. Geosci., 16(1):139-158, 2012.

[28] M. G. Larson and S. Zahedi. Stabilization of higher order cut finite element methods on surfaces. ArXiv e-prints, Oct. 2017.

[29] C. Lehrenfeld and A. Reusken. Analysis of a high-order unfitted finite element method for elliptic interface problems. IMA J. Numer. Anal., 38(3):1351-1387, 2018.

[30] A. Massing, M. G. Larson, A. Logg, and M. E. Rognes. A stabilized Nitsche fictitious domain method for the Stokes problem. J. Sci. Comput., 61(3):604-628, 2014.

[31] M. Miyashita and N. Saito. Hybridized discontinuous Galerkin method for elliptic interface problems: error estimates under low regularity assumptions of solutions. J. Sci. Comput., 76(3):1657$1673,2018$.

[32] I. Oikawa and F. Kikuchi. Discontinuous Galerkin FEM of hybrid type. JSIAM Letters, 2:49-52, 2010.

[33] M. A. Olshanskii, A. Reusken, and J. Grande. A finite element method for elliptic equations on surfaces. SIAM J. Numer. Anal., 47(5):3339-3358, 2009.

[34] C. H. Villanueva and K. Maute. CutFEM topology optimization of 3D laminar incompressible flow problems. Comput. Methods Appl. Mech. Engrg., 320:444-473, 2017.

\section{Authors' addresses:}

Erik Burman,

e. burman@ucl.ac.uk

Daniel Elfverson,

daniel.elfverson@umu.se

Peter Hansbo,

peter.hansbo@ju.se

Mats G. Larson,

mats.larson@umu.se

Karl Larsson,

karl.larsson@umu.se

Mathematics, University College London, UK

Mathematics and Mathematical Statistics, Umeå University, Sweden

Mechanical Engineering, Jönköping University, Sweden

Mathematics and Mathematical Statistics, Umeå University, Sweden

Mathematics and Mathematical Statistics, Umeå University, Sweden 